\title{
Evaluation of Retrograde Intrarenal Surgery Using Semirigid Ureterorenoscope in Treatment of Renal Calculi- A Prospective Study
} Dr. Pranay Patel ${ }^{1}$, Dr. Rajeev Chaudhari ${ }^{2}$, Dr. Tarun Singh ${ }^{3 *}$, Dr. Rishikesh A Kore ${ }^{4}$, Dr. Priyank M Shah ${ }^{5}$, Dr. Satyadeo Sharma ${ }^{6}$

\author{
${ }^{1}$ DNB Urology Resident, Ruby Hall Clinic, Pune, Maharashtra 411001, India \\ ${ }^{2} \mathrm{MCH}$ (Urology), Head of Department Urology, Ruby Hall Clinic, Pune Maharashtra 411001, India \\ ${ }^{3}$ DNB Urology Resident, Ruby Hall Clinic, Pune, Maharashtra 411001, India \\ ${ }^{4}$ DNB Urology, Ruby Hall Clinic, Pune, Maharashtra 411001, India \\ ${ }^{5}$ DNB Urology, Ruby Hall Clinic, Pune, Maharashtra 411001, India \\ ${ }^{6}$ DNB Urology, Ruby Hall Clinic, Pune, Maharashtra 411001, India
}

Introduction and Objective: Urolithiasis is one of the most common urological disorder today. Though PCNL is the gold standard treatment for large renal stones, Semirigid or flexible RIRS is evolving as a treatment modality for fair sized renal calculi up to $2-2.5 \mathrm{~cm}$. The objectives of this study are to assess usefulness, effectiveness and complications of RIRS using semi rigid ureterorenoscope in treatment of urolithiasis. Methods: This is hospital based prospective, observational study of 30 patients of renal calculi sized $>1.5 \mathrm{~cm}$ and $<3 \mathrm{~cm}$. They were willing to undergo RIRS with semirigid ureterorenoscope at the beginning. Later if required, they underwent PCNL. ESWL was given later after 3 weeks, if required. Extent of stone clearance and complications were recorded. All patients were followed up at 3 weeks and 3 months to check for residual stones. Results: The major advantages of semirigid ureterorenoscopes are stone free rate of $33.33 \%$ without any additional ESWL, good vision, better accessibility, long instrument stability and low incidence of complications (mild UTI 3.33\%). Follow up along with ESWL, additional stone clearance rate was $30 \%$. So the total stone clearance rate of sRIRS with or without ESWL was $63.33 \%$. Remaining 11(36.67\%) were converted to PCNL due to poor or non visibility of stone. In these patients, 8 cases (26.67\%) achieved complete stone clearance and in 3 cases $(10 \%)$ additional ESWL was required. Conclusion: RIRS with semirigid ureterorenoscope is effective, safe and alternate treatment modality to PCNL. sRIRS is completely successful in one third of patients and combined with ESWL, complete stone clearance is achieved in two third of the patients. Surprisingly, the grade of hydronephrosis, internal architectures of PC system and angulation at PUJ has no correlation with the outcome.

Keywords: ESWL, PCNL, RIRS, Renal calculi, Semirigid ureterorenoscope.

Copyright $(\odot 2021$ The Author(s): This is an open-access article distributed under the terms of the Creative Commons Attribution 4.0 International License (CC BY-NC 4.0) which permits unrestricted use, distribution, and reproduction in any medium for non-commercial use provided the original author and source are credited.

\section{INTRODUCTION}

In today's world, Urolithiasis is the most common urological disorder. Many factors play a role in incidence as well as prevalence of renal calculi like geographical region, age, gender, race, dietary factors, environmental factors and socioeconomic status etc [1]. Treatment for renal stones range from medical management to various surgical procedures. Surgical procedures include traditional open surgeries to modern minimal access procedures [2, 3]. The minimally invasive procedures include extracorporeal shockwave lithotripsy (ESWL), ureterorenoscopy (URS), retrograde intrarenal surgery (RIRS), percutaneous nephrolithotomy (PCNL) etc [3]. ESWL disintegrates stones in the kidney and upper urinary tract through the use of shock waves with limited success rate [3]. PCNL is through a direct access to the pelvicalyceal system under guidance of fluoroscopy or ultrasound [4]. RIRS involves introduction of endoscope, either rigid, semi rigid or flexible, through urethral meatus in retrograde fashion into pelvicalyceal system. These two procedures are found to have good success rate [5]. PCNL has established as the gold standard treatment for large renal calculi $(>1.5 \mathrm{~cm})$. RIRS is an evolving treatment modality in case of smaller sized calculi [6]. Both the procedures are technically demanding. These procedures are also expensive. Nowadays, flexible 
ureterorenoscopes are most commonly used for RIRS. The major advantages of flexible ureterorenoscopes are better success rate, better stone free rate, fair vision, better accessibility and low incidence of complications. The disadvantages include longer operative time, more incidence of postoperative fever, septicemia, high cost to the patients, need of more expertise, lack of long durability of the equipment etc [7-11]. In such circumstances, sRIRS can prove to be beneficial in treatment of renal calculi.

In our study, we are going to assess usefulness and effectiveness of sRIRS, in treatment of renal calculi. Effectiveness of this method will be studied in terms of stone clearance rate, operative time, duration of stay, incidence of complications, anatomy of pelvicalyceal system, associated problems of the pelviureteric junction, dilatation of pelvis or ureter, stone factors (size, shape, volume, density and composition of stone), clearance of the stone, associated postoperative complications and treatment for residual calculi if any.

\section{Materials and Methods}

This was a hospital based prospective, observational study in which total 30 patients of renal calculi were selected. At the beginning of surgery, they were willing to undergo RIRS with semirigid ureterorenoscope first. Later if required, they underwent PCNL. They were assessed after 3 weeks post operatively. ESWL was given for residual calculi after 3 weeks. Study was conducted during March 2016 to June 2018 after ethical permission of institutional ethical committee.

Inclusion criteria for study participants was patients with diagnosis of renal calculi of size more than $1.5 \mathrm{~cm}$ and less than $3.0 \mathrm{~cm}$. Exclusion criteria were patients with calculi located at other than renal pelvis and upper calyx, calculi size less than $1.5 \mathrm{~cm}$ or more than $3.0 \mathrm{~cm}$ and patients with age $<18$ years.

The study population comprised of patients with renal calculi was conducted in department of urology, Ruby Hall Clinic, Pune, Maharashtra. The patients who were diagnosed with renal calculi, were subjected to radiological studies including plain X-ray KUB, ultrasonography (USG) of abdomen and pelvis, intravenous pyelography (IVP) or CT Urography. DTPA renal scan was done as and when required. The facts studied include associated PUJ problems like narrow PUJ or kink, angle of calyces with ureter, renal pelvis being intrarenal or extra renal, dilated or not dilated renal pelvis, associated hydroureter present or not and stone indices like number, size, volume, shape and density in terms of Hounsfield units (HU). Stone volume (in cubic millimetres) was calculated using formula (Volume $=0.523 \mathrm{X}$ Length X Width X Height of stone in millimetres) [12]. All the routine investigations and pre-anaesthetic checkup was done. Written informed consent had been taken from all the patients. Total 30 patients were included in the study, who had undergone sRIRS or PCNL after non visualization of stone in sRIRS. Fragmentation was not initiated in sRIRS if PCNL was planned.

Under proper anaesthesia, a terumo guidewire of size 0.035 inch is put in the ureter to allow safe passage of a $6 / 10 \mathrm{~F}$ Nottingham ureteric dilator to achieve ureteric dilatation. Retrograde pyelography (RGP) was done first during procedure and findings were noted. Depending on RGP \& IVP / CT Urography images, angle formed by each calyx with ureter was studied. Before sRIRS, Inj.Hyoscine Butylbromide 20 $\mathrm{mg}$ was given IV. The ureteric dilatation was done up to 10f over a guide wire up to PUJ. RIRS was done with Richard Wolfe 6.5-8.5F, $5^{\circ}$ semirigid ureterorenoscope .The stone is visible or not, complete or partial, its percentage of visibility was recorded. Perioperative findings such as size, number, nature and location of stone were recorded. Calculi were fragmented by holmium-yttrium-aluminium-garnet (Ho:YAG) laser (Sphinx Jr., LISA lasers, USA) using a $365 \mu / 550 \mu$ laser fibre. The energy and frequency settings were adjusted as required. To improve visualization \& fragmentation, flank hand manipulation was used by Assistant. After the satisfactory fragmentation, fluoroscopy is done to look for residual stones, if any. At the end, a $6 \mathrm{~F} / 26 \mathrm{~cm}$ DJ stent is placed in the ureter in all the patients. Extent of stone clearance is recorded as complete or partial. Complications, if any, are noted and treated accordingly. In cases of failed sRIRS or poor visibility of stone, standard PCNL with pneumolithotripsy was done at the same time, if no intraoperative problem during sRIRS.

All the patients were followed up at 3 weeks postoperatively and 3 months after stent removal. At each follow up, a plain X-ray KUB and USG of abdomen and pelvis were done to look for any residual calculi. Patients with fragments of size $3 \mathrm{~mm}$ or less were considered as complete clearance. For any residual stones at 3 weeks, ESWL was given as and when required. Number of ESWL sessions required werenoted. The DJ stent was removed when both X-ray KUB and USG of abdomen and pelvis suggested complete stone clearance. The various findings noted were- success rate of sRIRS in terms of fragmentation rate, stone free rate, duration of surgery, incidence of complications and length of hospitalization. All the data collected from above observations was entered in a prescribed Data collection form for each patient and is tabulated in worksheet. The entire statistical analysis was performed using Statistical Package for Social Sciences (SPSS version 17.0, Chicago, IL) for MS Windows. 


\section{RESULTS}

Table-1

\begin{tabular}{|c|c|c|}
\hline Variables & & Number $($ Total $=30)$ \\
\hline \multirow[t]{2}{*}{ Gender } & Male & 19 \\
\hline & Female & 11 \\
\hline \multirow{6}{*}{ Age } & $18-30$ & 3 \\
\hline & $31-40$ & 7 \\
\hline & $41-50$ & 10 \\
\hline & $51-60$ & 5 \\
\hline & $61-70$ & 3 \\
\hline & $>70$ & 2 \\
\hline \multirow[t]{2}{*}{ Pelvis } & Intrarenal & 28 \\
\hline & Extra renal & 2 \\
\hline \multirow{6}{*}{$\begin{array}{l}\text { Extent of visibility } \\
\text { Of stone }\end{array}$} & $100 \%$ (complete) & 12 \\
\hline & 80 to $90 \%$ & 2 \\
\hline & 60 to $70 \%$ & 3 \\
\hline & 30 to $50 \%$ & 2 \\
\hline & 10 to $20 \%$ & 3 \\
\hline & $0 \%$ (no visibility) & 8 \\
\hline \multirow{4}{*}{ Procedure Done } & Right Srirs & 8 \\
\hline & Left sRIRS & 11 \\
\hline & Right PCNL & 7 \\
\hline & Left PCNL & 4 \\
\hline \multirow{3}{*}{ Complications } & No complications & 27 \\
\hline & Hemorrhage & 2 \\
\hline & UTI & 1 \\
\hline
\end{tabular}

Table-1 shows that urolithiasis had been more common in males $19(63.33 \%)$ patients than females with $11(36.67 \%)$ patients. Age group of 41-50 years had maximum number of patients with urolithiasis, Intrarenal pelvis was the most common type of anatomy in $28(93.33 \%)$ of patients in this group. Stone was completely visible in 12 patients (40\%), partially visible in 7 patients $(23.33 \%)$ and not visible in 8 patients (26.66\%). In $19(63.33 \%)$ patients, sRIRS was done with or without ESWL and in $11(36.67 \%)$ patients it was converted to PCNL. No major complication was seen except in 1 patient who had undergone sRIRS, had mild UTI and 2 patients who had PCNL required blood transfusion for hemorrhage.

Table-2

\begin{tabular}{|l|l|l|l|}
\hline & & USG & IVU/CT \\
\hline \multirow{3}{*}{$\begin{array}{l}\text { Size of calculus } \\
\text { in m) }\end{array}$} & $15-20$ & 15 & 12 \\
\cline { 2 - 4 } & $21-25$ & 8 & 9 \\
\cline { 2 - 4 } & $26-30$ & 7 & 9 \\
\hline Extent of hydronephrosis & Grade 0 & 7 & 7 \\
\cline { 2 - 4 } & Grade 1 & 16 & 12 \\
\cline { 2 - 4 } & Grade 2 & 1 & 1 \\
\cline { 2 - 4 } & Grade 3 & 6 & 10 \\
\hline
\end{tabular}

In Table-2, On Ultrasonography (USG) maximum 15 patients had stone size between $15-20 \mathrm{~mm}$ followed by 8 patients in 21-25 mm range. In 7 patients stone size was between $26-30 \mathrm{~mm}$. On IVU/CT scan findings, there was slight variation with 12 patients having size of $15-20 \mathrm{~mm} \& 9$ patients having the stone size of 21-25 mm.9 patients had $26-30 \mathrm{~mm}$ size stone on IVP/CT Urography. Grade 1 was the most common grade of hydronephrosis according to both USG and IVU or CT scan in 16 and 12 patients. 
Table-3

\begin{tabular}{|l|l|l|}
\hline Stone volume (in cmm) & $<1000$ & 3 \\
\cline { 2 - 3 } & $1001-2000$ & 9 \\
\cline { 2 - 3 } & $2001-3000$ & 13 \\
\cline { 2 - 3 } & $3001-4000$ & 4 \\
\cline { 2 - 3 } & $4001-5000$ & 1 \\
\hline \multirow{5}{*}{ Stone density(in HU) } & $401-800$ & 10 \\
\cline { 2 - 3 } & $801-1200$ & 12 \\
\cline { 2 - 3 } & $>1200$ & 8 \\
\hline
\end{tabular}

In Table-3, based on CT Urography findings, 12 patients had stone volume ranging from 900 $\mathrm{cmm}$ to $2000 \mathrm{cmm}$. In 17 patients, stone volume was between 2001 to $4000 \mathrm{cmm}$. In 1 patient it was 4110 $\mathrm{cmm}$. The mean +/- SD of stone volume was $2223.6+/-$ $953.1 \mathrm{cmm}$. Stone density was estimated in Hounsfield units (HU) as per CT scan findingsin allpatients. It ranged from 250 to $1400 \mathrm{HU}$. The mean +/- SD density in our study was $911.3+/-288.7$ HU. The immediate postoperative complete stone clearance was achieved in 11 patients of only sRIRS with complete stone clearance rate of $36.66 \%$. In other 8 patients, partial clearance was achieved who needed subsequent ESWL.

Table-4

\begin{tabular}{|l|l|l|l|}
\hline & & sRIRS & PCNL \\
\hline Duration of surgery & $<60$ & 7 & 0 \\
\cline { 2 - 4 } in minutes (as per OT records) & $61-90$ & 4 & 0 \\
\cline { 2 - 4 } & $91-120$ & 7 & 2 \\
\cline { 2 - 4 } & $121-150$ & 1 & 4 \\
\cline { 2 - 4 } & $>150$ & 0 & 5 \\
\hline \multirow{4}{*}{$\begin{array}{l}\text { (in days) } \\
\text { (ination of hospital stay }\end{array}$} & 1 & 10 & 0 \\
\cline { 2 - 4 } & 2 & 8 & 0 \\
\cline { 2 - 4 } & 3 & 1 & 1 \\
\cline { 2 - 4 } & 4 & 0 & 8 \\
\cline { 2 - 4 } & $>5$ & 0 & 2 \\
\hline No of ESWL sessions required & 1 & 2 & 1 \\
\cline { 2 - 4 } & 2 & 3 & 2 \\
\cline { 2 - 4 } & 3 & 4 & 0 \\
\hline
\end{tabular}

Duration of surgery was measured from getting the patient on table to patient shifted from the table at the end of surgery. The mean +/- SD duration of sRIRS surgery in 19 patients in our study was $87.37+/-30.52$ minutes. In patients in whom PCNL had to be done the mean duration of surgery was $148.18+/-20.88$ minutes. The overall duration was longer when PCNL was done due to sRIRS in the beginning. Amongst the 19 patients in whom only sRIRS was done, 10 patients were hospitalized for 1 day. In the same group, 8 patients were for2-day admission \& 1 patient stayed for 3 days. None of the patient of sRIRS had stay of longer than 3 days.
In 11 patients of PCNL, 8 patients had stay of 4 days, 2 patients of 5 days. Only 1 patient had short stay of 3 days. In patients who underwent sRIRS, the mean +/- SD duration of hospitalization was $1.53+/$ 0.61 days. When PCNL was done the mean $+/-$ SD stay had increased to $4.09+/-0.54$ days. Total 9 patients of sRIRS had residual calculi for which ESWL was given. A single session of ESWL cleared all fragments in 2 patients. 3 patients of only sRIRS required 2 sessions and 3 sessions were given in 4 patients.

Table-5

\begin{tabular}{|l|l|l|l|l|}
\hline \multirow{2}{*}{ Follow up } & \multicolumn{3}{|l|}{$\mathbf{1}^{\text {st }}$ follow up (3 weeks) } & \multicolumn{2}{l|}{$\mathbf{2}^{\text {nd }}$ follow up (3 months) } \\
\cline { 2 - 5 } & sRIRS & PCNL & sRIRS & PCNL \\
\hline Residual calculi (>3mm) & 9 & 3 & 0 & 0 \\
\hline Residual calculi $(\leq 3 \mathrm{~mm})$ & 0 & 4 & 3 & 1 \\
\hline No residual calculi & 10 & 4 & 16 & 10 \\
\hline Total & 19 & 11 & 19 & 11 \\
\hline
\end{tabular}


As shown in Table-5, out of 19 patients of only sRIRS, 10 patients were completely clear of any residual fragments. Thus, complete stone clearance rate at 1 st follow up was $33.33 \%$. 9 patients had residual calculi which required ESWL for complete clearance.

7 patients who underwent PCNL had residual fragments. 3 of this post PCNL group required ESWL since they had residual stones $>3 \mathrm{~mm}$ size at 3 weeks follow up visit. 3 patients of sRIRS and 1 patient of PCNL, hadresidual calculi of $\leq 3 \mathrm{~mm}$, respectively at second follow up after 3 months.

\section{DisCUSSION}

In our study, the mean age +/- SD was 47.43 and 13.34 years respectively. The mean age $+/-\mathrm{SD}$ in Gokhan Atis et al., study was $42.8+/-11.5$ years [13]. Thus, the age incidence matches with other studies conducted on the same topic. The male to female ratio in our study was 1.78:1 when compared to Gokhan Atis et al., ratio of 1:1.78 [12]. In Iraklis C. Mitsogiannis et $a l$., the ratio was $2: 3$ [13]. The findings of our study have shown a male preponderance which was in contrary to studies by Gokhan Atis et al., and Iraklis C. Mitsogiannis et al., where they have found female preponderance $[12,13]$.

In our study, the mean $+/-$ SD size of calculi on ultrasonographic examination was $21.57+/-5$. $1 \mathrm{~mm}$ and on CT/IVU it was $22.13+/-4.9 \mathrm{~mm}$. The mean +/SD stone size in Gokhan Atis et al., was $15.10+/-5.70$ mm12. In Piotr Bryniarski et al., it was $2.4+/-1.1 \mathrm{cms}$ in RIRS group and $24+/-8 \mathrm{~mm}$ in PCNL group [14]. In Gyoo Hwan Jung et al., it was $23.7+/-6.4 \mathrm{~mm}$ in PCNL group and $20.0+/-4.1 \mathrm{~mm}$ in RIRS group using flexible ureteroscope [15]. The stone sizes are nearly similar in all the studies including our study [12, 14, 15]. The mean grade of hydronephrosis in 16 patients of sRIRS group, was $2.062+/-1.124$. In 7 patients of PCNL group, it was $1.714+/-0.951$. This difference in grade of hydronephrosis in patients undergoing sRIRS or PCNL was not significant $(\mathrm{P}$ value $=0.4838$ ). Thus presence or absence of hydronephrosis is of no value to predict the success or failure of sRIRS.

In our study, 28 patients $(93.33 \%)$ had intrarenal pelvis and 2 patients $(6.67 \%)$ had extrarenal pelvis. The mean $+/-$ SD of stone volume was 2223.6 $+/-953.1 \mathrm{cmm}$. In Igor Sorokin et al study, stone volume was in the range of 150.7 to $644.7 \mathrm{cmm}$. They concluded that the stone volume has large impact on operative time and operative time increases roughly by 2 minutes per $100 \mathrm{cmm}$ increase in stone volume [16]. In study by Gyoo Hwan Jung et al., the mean stone volume in PCNL group was $1853.6+/-1187.1 \mathrm{cmm}$. In RIRS group using flexible ureteroscope it was 1491.5 +/- $1384.1 \mathrm{cmm}$ [15]. In our study, the mean +/- SD stone density was $911.3+/-288.7 \mathrm{HU}$ where Igor Sorokin et al., study, it was $936.4+/-343.3$ HU. They found that stone density had no significant impact on operative time [16]. Stone was visible satisfactorily $63.33 \%$ in our study. When only edge of the stone was seen, it was declared as $<25 \%$ of visibility. In $26.67 \%$ patients, stone could not be seen at all. In these cases, on CT scan/IVP, it appeared to be a straight and easily accessible preoperatively. The mean visibility was $36.67 \%$ in patients in whom PCNL was done. The difference in these values is statistically significant $(\mathrm{P}$ value $=<0.0001)$. Thus extent of stone visibility had direct association with success of sRIRS. The stone accessibility rate in Khaled Mursi et al., was in $83 \%$ of the patients [17]. In the study by Iraklis C. Mitsogiannis et al., it was $85 \%$ in similar cases [13]. Thus, in our study stone visibility rate was less.

Right sRIRS was done in 15 patients and left sRIRS in 15 patients. Right sRIRS was done satisfactorily in 8 patients. In 7 patients, it failed due to non or poor visualization of stone, in whom PCNL was done. Left sRIRS was done satisfactorily in 11 patients. In 4 patients, it failed, in whom PCNL was done. PCNL was done 8 patients. In a study by Gokhan Atis et al., in 13 patients $(52 \%)$ left side was operated and 12 patients (48\%) right side was operated [12]. In study by Piotr Bryniarski et al., 14 (43.7\%) were right sided and 18 $(56.3 \%)$ were left sided [14]. In 19 patients $(63.33 \%)$ we were able to reach the calculi with the semirigid ureteroscope, remaining 11 patients the stone was either not visible /poorly visible or not accessible in whom PCNL was done. Thus, the complete stone clearance rate of $36.66 \%$ was achieved, In other 8 patients partial clearance was achieved with mean clearance rate of $67.5+/-16.69 \%$. These patients had subsequent ESWL for clearance of residual stones. In a study by Gokhan Atis et al., on evaluation on 1 st postoperative day, clearance was $72 \%$. After 1 month of surgery it was $76 \%$ [12]. In Piotr Bryniarski et al., on first postoperative day 16 out of 32 patients $(50 \%)$ had residual fragments while 3 weeks after discharge 8 patients $(25 \%)$ had residual stones. In their study, PCNL group, 6 out of 32 patients $(18.75 \%)$ had residual fragments on first postoperative day and after 3 weeks only 2 patients $(6.25 \%)$ had residual fragments. Thus, the efficacy of PCNL was $82 \%$ and $94 \%$ on postoperative 1st day and 3 weeks respectively [14]. Iraklis Mitsogiannis et al., study, the stone free rate in 17 patients was $70.6 \%$ at the 1 st postoperative day and $82.3 \%$ after 1 month. ${ }^{13}$ The success rate in study of Khaled Mursi et al., was 53\% [17]. Thus success rate of complete clearance only with sRIRS in our study was $33.33 \%$ which was lower than studies by Piotr Bryniarski et al., (50\%), Khaled Mursi et al., (53\%), Gokhan Atis et al., (72\%) and Iraklis C. Mitsogiannis et al., $(70.6 \%)$.

Out of total 30 patients, in 19 or $63.33 \%$ of patients, we could reach renal pelvis, visualize and fragment the calculi well. In 11 patients $(36.67 \%)$ sRIRS could not be done satisfactorily and PCNL had to be done. In Gokhan Atis et al., they were able to 
fragment the calculi in $53 \%$ of patients whereas in $47 \%$ they were not able to reach the pelvis or calculi [12]. Iraklis Mitsogiannis et al., had failed sRIRS in 3 patients out of 20 [13]. In a study by Khaled Mursi et al., 4 out of 15 patients had access failure [17]. The mean $+/$ - SD duration of sRIRS surgery in 19 patients in our study was $87.37+/-30.52$ minutes. When PCNL was done after failed sRIRS, mean $+/$ - SD duration was $148.18+/-20.88$ minutes. The mean +/- SD duration in Gokhan Atis et al., was $71.9+/-17.9$ minutes [12]. Piotr Bryniarski et al., was $85+/-17.2$ minutes [14], In study by Iraklis Mitsogiannis et al., it was $69.4 \mathrm{~min}$ with range of 37.2 to $94.5 \mathrm{~min}$ [13]. The surgery duration in our study is slightly more than studies by GokhanAtis et al., Piotr Bryniarski et al., and Iraklis C. Mitsogiannis et al., since it was measured from getting patient on table to patient shifted from the table.

The mean +/- SD duration of hospitalization was $1.53+/-0.61$ days. With PCNL it increased to 4.09 +/-0.54 days compare with semirigid URS, in Gokhan Atis et al., it was 1.5 +/- 1.2 days [12], Piotr Bryniarski et al., was $6.8+/-3.4$ days and in PCNL group it was $11.3+/-4.4$ days [14] and in IraklisMitsogiannis et al., it was 1.4 days (Range 1.1-4.3 days) [13]. The duration of hospitalization in our study in sRIRS group was comparable with the studies by Gokhan Atis et al., Iraklis C. Mitsogiannis et al., and is much less than in study by Piotr Bryniarski et al., ESWL was given to 9 patients of sRIRS and in 3 patients of PCNL for residual calculi. It was observed that clearance of residual calculi becomes much easier due to already fragmented stone. Iraklis Mitsogiannis et al., required ESWL in 3 patients $(17.65 \%)$ [13]. The mean numbers of sessions were 1.5. No major complication had occurred in our study. Only 1 patient $(3.33 \%)$ had mild urinary tract infection. 2 patients $(6.67 \%)$ with PCNL required blood transfusion. Thus a $3.33 \%$ complication rate was observed in our study as compared to $16 \%$ in study by Gokhan Atis et al., [12]. In study by Piotr Bryniarski et al., 1 out of 32 patients $(3.1 \%)$ required blood transfusion. In their study fever was seen in 8 patients $(25 \%)$ of sRIRS group and $9(28.1 \%)$ of PCNL group [14]. Iraklis Mitsogiannis et al., reported fever in 2 patients (10\%) [13]. Thus sRIRS is much safer approach when it is feasible.

The complete stone clearance rate at 1 st follow up after 3 weeks was $33.33 \%$. We started ESWL sessions only after assessment at $1^{\text {st }}$ follow up. At 1 month follow up, Gokhan Atis et al., stone clearance rate was 76\% [12] and Iraklis Mitsogiannis et al., the stone free rate was $82.3 \%$ [13]. As we have used ESWL for residual calculi, we are able to clear the residual fragments in all at the 2nd follow up (after 3 months).Thus the total clearance rate with sRIRS with or without ESWL was $63.33 \%$.

\section{Conclution}

sRIRS with or without ESWL is good treatment option for renal calculi, due to long stability of sRIRS. It is a cheap alternative without any significant complications. It is also feasible nowadays due to availability of lasers converting stone into powder for easy clearance. sRIRS is a good alternative to avoid more invasive \& technically demanding treatment modality like PCNL. The grade of hydronephrosis, internal architectures of PC system and angulation at PUJ was found to have no significant correlation with the expected visibility \& accessibility of stone. Certain technical modifications do help is better visibility and fragmentation during sRIRS. It is completely successful \& gives complete stone clearance in two third of the patients when combined with ESWL.

Funding: No funding sources

Conflict of interest: None declared

Ethical approval: The study was approved by the Institutional Ethics Committee.

\section{REFERENCES}

1. Trinchieri A. Epidemiology of urolithiasis: an update. Clin cases Miner bone Metab. 2008 May;5(2):101-6.

2. Geavlete P, Georgescu D, NiţĂ G, Mirciulescu V, Cauni V. Complications of 2735 Retrograde Semirigid Ureteroscopy Procedures: A SingleCenter Experience. J Endourol. 2006 Mar;20(3):179-85.

3. Srisubat A, Potisat S, Lojanapiwat B, Setthawong V, Laopaiboon M. Extracorporeal shock wave lithotripsy (ESWL) versus percutaneous nephrolithotomy (PCNL) or retrograde intrarenal surgery (RIRS) for kidney stones (Review). Cochrane Libr. 2014;(11):1-41.

4. Lojanapiwat B. The ideal puncture approach for PCNL: Fluoroscopy, ultrasound or endoscopy? Indian J Urol. 2013 Jul;29(3):208-13.

5. Chibber P. Retrograde intra-renal surgery: Where are we today? Indian J Urol. 2008 Oct;24(4):509.

6. Prabhakar M. Retrograde ureteroscopic intrarenal surgery for large $(1.6-3.5 \mathrm{~cm})$ upper ureteric/renal calculus. Indian J Urol. 2010;26(1):46-9.

7. Voigt K, de Jong T. How do the three modalities for stone removal (PCNL, URS and ESWL) compare to each other with respect to complication rate, stone-free rate and operation time/ costs? [Internet]. [cited 2016 Oct 18]. p. 1-5. Available from:

https://www.researchgate.net/publication/2714423 75_How_do_the_three_modalities_for_stone_rem oval_PCNL_URS_and_ESWL_compare_to_each_ other_with_respect_to_complication_rate_ston efree_rate_and_operation_time_costs 
8. Yencilek F, Sarıca K, Gürpınar T, Göktaş C, Cangüven Ö, Albayrak S. A comparison of shock wave lithotripsy, semirigid and flexible ureteroscopy in the management of proximal ureteral calculi. Turkish J Urol. 2009;35(2):101-7.

9. Akman T, Binbay M, Ozgor F, Ugurlu M, Tekinarslan E, Kezer C, Aslan R, Muslumanoglu AY. Comparison of percutaneous nephrolithotomy and retrograde flexible nephrolithotripsy for the management of 2-4 cm stones: a matched- pair analysis. BJU International-British Journal of Urology. 2012 May 1;109(9):1384-1389.

10. ElGanainy E, Hameed DA, Elgammal M, AbdElsayed AA, Shalaby M. Experience with impacted upper ureteral Stones; should we abandon using semirigid ureteroscopes and pneumatic lithoclast?Int Arch Med. 2009;2(1):13.

11. Khairy-Salem H, El Ghoneimy M, El Atrebi M. Semirigid Ureteroscopy in Management of Large Proximal Ureteral Calculi: Is There Still a Role in Developing Countries? Urology. 2011 May;77(5):1064-8.

12. Atis G, Gurbuz C, Arikan O, Canat L, Kilic M, Caskurlu T. Ureteroscopic Management with Laser Lithotripsy of Renal Pelvic Stones. J Endourol. 2012 Aug;26(8):983-7.

13. C Mitsogiannis I, Papatsoris A, Varkarakis J, Skolarikos A, Deliveliotis C. Semirigid Laser
Ureterolithotripsy for Single Large Renal Pelvic Stones. Med Surg Urol. 2013;S4:2-4.

14. Bryniarski P, Paradysz A, Zyczkowski M, Kupilas A, Nowakowski K, Bogacki R. A Randomized Controlled Study to Analyze the Safety and Efficacy of Percutaneous Nephrolithotripsy and Retrograde Intrarenal Surgery in the Management of Renal Stones More Than $2 \mathrm{~cm}$ in Diameter. J Endourol. 2012 Jan;26(1):52-7.

15. Jung GH, Jung JH, Ahn TS, Lee JS, Cho SY, Jeong CW, Lee SB, Kim HH, Oh SJ. Comparison of retrograde intrarenal surgery versus a singlesession percutaneous nephrolithotomy for lowerpole stones with a diameter of 15 to $30 \mathrm{~mm}$ : A propensity score-matching study. Korean journal of urology. $2015 \mathrm{Jul} ; 56(7): 525$.

16. Sorokin I, Cardona-Grau DK, Rehfuss A, Birney A, Stavrakis C, Leinwand G, Herr A, Feustel PJ, White MD. Stone volume is best predictor of operative time required in retrograde intrarenal surgery for renal calculi: implications for surgical planning and quality improvement. Urolithiasis. 2016 Nov;44(6):545-50.

17. Mursi K, Elsheemy MS, Morsi HA, Ali Ghaleb AK, Abdel-Razzak OM. Semi-rigid ureteroscopy for ureteric and renal pelvic calculi: Predictive factors for complications and success. Arab J Urol. 2013 Jun;11(2):136-41. 